\section{Simulation of Shish-Kebab Crystallite Induced by a Single Prealigned Macromolecule}

\section{Wenbing Hu, ${ }^{\dagger}$ Daan Frenkel, ${ }^{*, \dagger}$ and Vincent B. F. Mathot ${ }^{\ddagger}$}

FOM Institute for Atomic and Molecular Physics, Kruislaan 407, 1098 SJ Amsterdam, The Netherlands, and DSM

Research, P.O. Box 18 Geleen, The Netherlands

Received May 23, 2002

Revised Manuscript Received J une 28, 2002

Crystal nucleation is an activated process. In a supersaturated solution, small crystallites may dissolve again. Crystals can only be formed when a (rare) fluctuation leads to the formation of a crystallite with a size that exceeds a threshold. The probability to form such a "critical" crystal nucleus is determined by its excess free energy. F or a simple molecular crystal, ${ }^{1}$ the free energy change for forming a small crystallite from the liquid is approximately given by

$$
\Delta \mathrm{F}(\mathrm{n})=-\mathrm{n}|\Delta \mu|+\mathrm{A} \gamma
$$

where $\mathrm{n}$ is the number of particles in the nucleus and $\Delta \mu$ is the difference between the chemical potentials of the particles in the (metastable) liquid phase and in the (stable) crystal phase. The surface area of the crystallite is denoted by $A$, and the solid-liquid interfacial free energy is denoted by $\gamma$. The free energy of a crystal nucleus contains two competing contributions: a (negative) bulk term favoring the formation of crystallites is counteracted by a (positive) surface term. For small crystallites, the surface term dominates, but for larger crystallites the bulk term becomes more important.

In the nucleation of chainlike polymers, we must consider the anisotropy introduced by the shape of the molecules. Assuming that a bundle of chain stems form a cylindrical polymer crystallite with a radius $r$ and a thickness I, the free energy change for forming such a crystallite becomes

$$
\Delta \mathrm{F}(\mathrm{n})=-\pi \mathrm{r}^{2}|| \Delta \mu \mid+2 \pi \mathrm{r}^{2} \gamma_{\mathrm{e}}+2 \pi \mathrm{r} \mathrm{\gamma}_{1}
$$

where $n=\pi r^{2}$ is the total number of units in the crystallite; $\gamma_{\mathrm{e}}$ and $\gamma_{1}$ are the surface free energies corresponding to the stem ends and the lateral surface, respectively. The most favorable size of critical nucleus can be cal culated from $\partial \Delta \mathrm{F}(\mathrm{n}) / \partial \mathrm{r}=\partial \Delta \mathrm{F}(\mathrm{n}) / \partial \mathrm{l}=0$ as $\mathrm{I}_{\text {crit }}$ $=4 \gamma_{\mathrm{e}} /|\Delta \mu|$ and $\mathrm{r}_{\text {crit }}=2 \gamma_{\mathrm{I}} /|\Delta \mu|$. Note that as the lateral surface free energy can be smaller by 1 order of magnitude than that of the stem-end surface of polymer crystallites, the critical thickness should belarger by 1 order of magnitude than the critical radius of nucleus. In other words, the most favorable shape of critical nuclei will be fiberlike. ${ }^{2}$ This shape requires a large degree of chain alignment, which is a very rare event in thermal fluctuations for an isotropic liquid, especially at high temperatures. As the supercooling is increased, the critical nucl eus becomes smaller and less el ongated. Therefore, polymer nucleation usually occurs via chain folding and requires a much higher degree of supercool-

\footnotetext{
† FOM Institute for Atomic and Molecular Physics.

‡ DSM Research.
}

ing than is needed to induce crystallization of simple molecular crystals.

There are several methods to increase the rate of polymer nucleation. All methods rely in one way or another on reducing the free energy barrier of crystal formation. The simplest method would be to decrease the temperature $T$ and thereby increase the thermodynamic driving force for crystallization. However, from a practical point of view this is often not a viable option-for instance, the polymer liquid may vitrify on cooling and results in poor crystallinity. A more practical method is to facilitate the incorporation of polymer chains in a crystallite by prealigning and stretching them in a flow field (extensional or shear). The rationale behind this approach is that aligning the chains in the fluid leads to a reduction of conformational entropy of the liquid polymers and hence to an increased thermodynamic driving force for crystallization. In practice, some prealigned long chains may induce the epitaxial crystallization of other chains. Shish-kebab crystallites, a special kind of polymer assembly, are frequently observed under these circumstances. ${ }^{3-7}$ Such crystallites exhibit a heterogeneous structure: a stack of foldedchain lamellae that form around a central fiber. Assemblies of this kind were first observed as row structures in crystallization of polyethylene from the melt under stress. ${ }^{8}$ Another practical method to enhance polymer nucleation is to use a nucleation agent, which is expected to decrease the surface free energy barrier by introducing a foreign substrate for heterogeneous nucleation. The most effective nucleation agents for polymers are usually those that act as a substrate for epitaxial growth. ${ }^{9-11}$

Epitaxial crystallization is clearly an important mechanism to accelerate polymer nucleation. But as discussed above, different scenarios are possible. One is that uniform prealignment of polymers due to at least one-dimensional match of crystal lattice on a substrate enhances the driving force for crystallization, i.e., the bulk term in eq 1. A second is that the substrate, on which the conventional layer-by-layer growth of polymer crystals takes place, acts as an agent for heterogeneous nucleation. In this case, where the central thread surface plays a role to decrease the surface free energy of critical nuclei, i.e., the surface term in eq 1 , an obvious question arises whether there is a lower limit of the central thread thickness for shish-kebab morphology.

Here, we present simulations to examine the effect of the alignment of a single chain in a polymer solution on the subsequent crystallization process. The aligned chain can be considered to be the prototypical result of shearing the fluid. But, as only one chain has been prealigned, any subsequent crystallization must be due to the primary nucleation induced by this "template".

In our simulations, we embedded a single aligned chain, spanning the periodic simulation box, in a semidilute solution of short polymer coils (see Figure 1). Initially, we prepared the sample system at infinitely high temperature, where the polymer solution is effectively athermal. Then we quenched the temperature to a value below the freezing curve, but above the temperature where homogeneous crystal nucleation could be observed on the time scale of our simulations. Since the lateral extent of the "template" is small, it can 


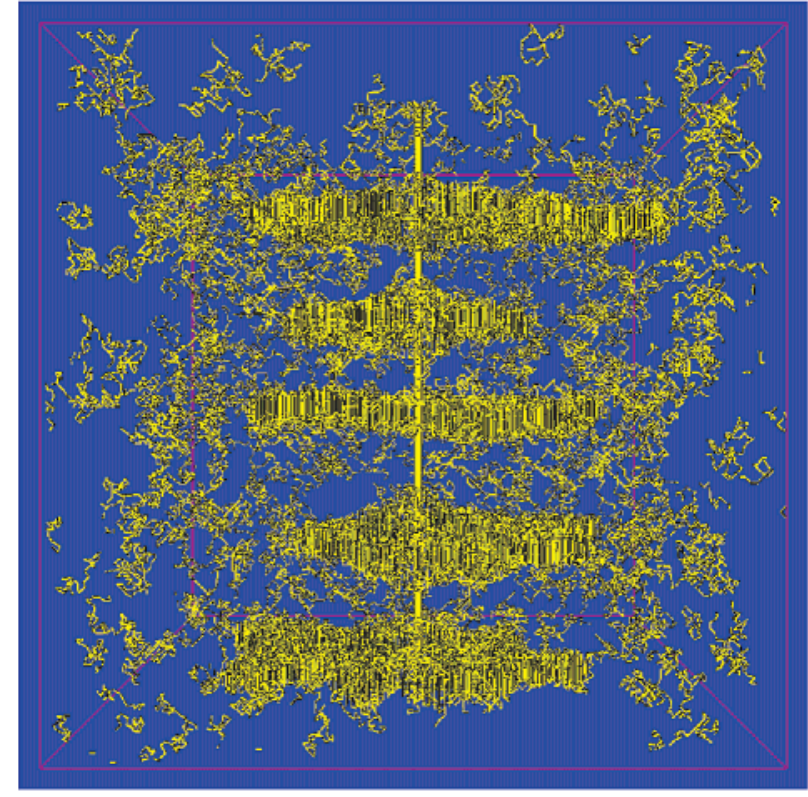

Figure 1. Snapshot of shish-kebab crystallite in a system containing 3840 chains, each having 32 units in a $128 \times 128$ $\times 128$ cubic lattice box with periodic boundary conditions. This corresponds to a volume concentration of 0.0586 . The fully aligned single-chain template has the same thickness as the other chains but has been drawn thicker to enhance visibility. The microrelaxation model ${ }^{12,13}$ performs single-site jumping plus partial sliding diffusion along the chain if necessary and uses M etropolis sampling with a Boltzmann weight $\left(c E_{c}+p E_{p}\right.$ $+b B) /\left(k_{B} T\right)$, where $c$ is the net change in the number of noncollinear connections of two consecutive bonds along the chain with potential energy change $E_{c}, p$ is the change in the number of nonparallel lateral packings of two bonds with $E_{p}$, and $b$ is the change in the number of chain-solvent contacts with energy $B$. In the simulations, $E_{p} / E_{c}$ is set to 1 to reflect semiflexibility of chains, $B / E_{C}$ is set to zero to avoid liquidliquid demixing, and $E_{d}\left(k_{B} T\right)$ is set to 0.4 , just above the temperature at which the homogeneous nucleation occurs. The snapshot was taken at time $1.18 \times 10^{5} \mathrm{MC}$ cycles, where one Monte Carlo (MC) cycle is defined as one microrelaxation jump of each chain unit on average.

only affect the very earliest stages of crystallization. To our surprise, we observed the formation of a row of crystal nuclei along the stretched chain. These nuclei subsequently grew to form a stack of lamellae with "shish-kebab" morphology. A snapshot is shown in Figure 1.

In Figure 1, the fold surfaces of lamellae are quite smooth and normal to the chain axis, and the crystallite thickness is almost constant at about seven chain units, which is considerably shorter than the chain length (32 units). We can directly observe the regularly folded conformation of the chains generally distributed in the lamellae, the structure suggested 45 years ago. ${ }^{14}$

The primary nucleation is determined by $n_{\text {crit, }}$, the size of the critical nucleus calculated from $\partial \Delta F(n) / \partial n=0$. A bove $n_{\text {crit, }}$ the crystal growth requires $\partial \Delta F(n) / \partial n<0$. Since $\gamma_{1} \ll \gamma_{\mathrm{e}}$ and at late stages of crystal growth $I \ll r$, the term of $2 \pi \mathrm{rl} \gamma_{1}$ can be omitted from eq 2 . Then, we get

$$
\Delta \mathrm{F}(\mathrm{n})=-\mathrm{n}|\Delta \mu|+2 \mathrm{n} \gamma_{\mathrm{e}} \mathrm{l}
$$

Equation 3 and the condition $\partial \Delta \mathrm{F}(\mathrm{n}) / \partial \mathrm{n} \leq 0$ give the growth thickness $I \geq 2 \gamma_{\mathrm{e}} /|\Delta \mu| \equiv I_{\min }$, a mi nimum value. $\Delta \mu$ depends on the concentration of polymers in solution. However, the concentration effect is relatively small,

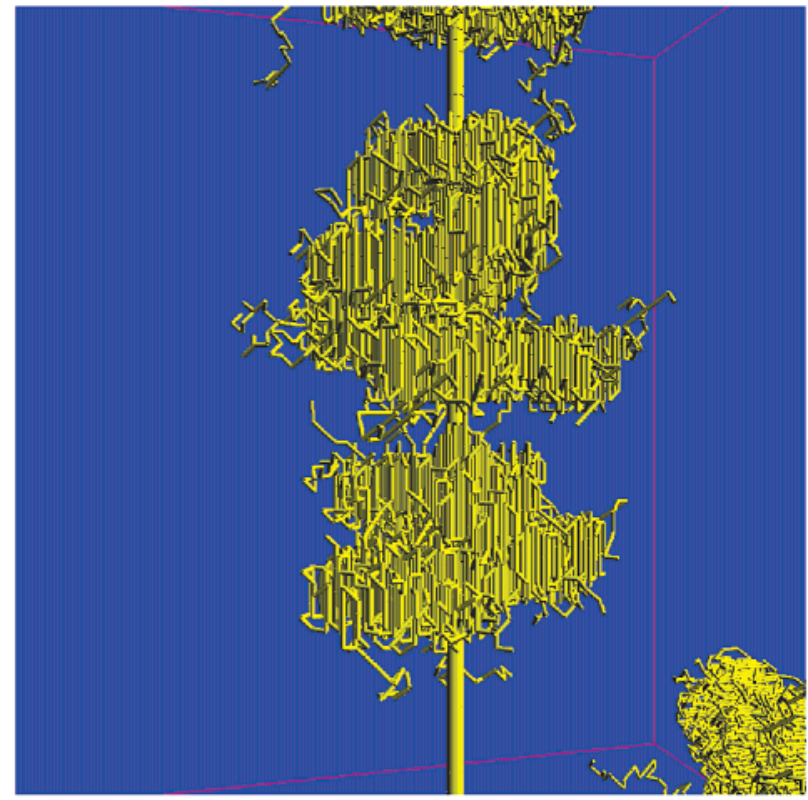

a.

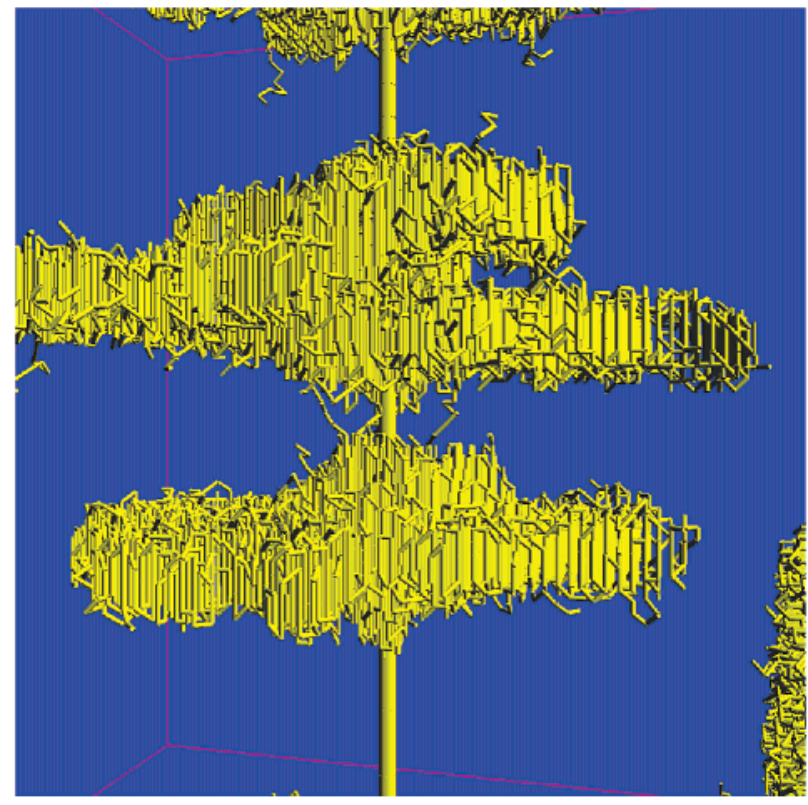

b.

Figure 2. Snapshot of a helicoi dal shish-kebab crystall ite for the same sample system (a) at a time $1.0 \times 10^{4} \mathrm{MC}$ and (b) at a time $5.0 \times 10^{4} \mathrm{MC}$ cycles with a slightly lower temperature $E_{d}\left(k_{B} T\right)=0.42$ than in Figure 1. Only the borders of cubic box and the chains in crystallites and the template (thick cylinder) are shown. At the slightly lower temperature, homogeneous nucleation happens to generate the lamella at the bottom-right corner of the figure.

and indeed, the lamellae grown with constant thickness are observed in Figure 1 even though the concentration in solution is somewhat lowered as chains become incorporated in lamellae.

The present expression for $I_{\min }$ is the same as that resulting from a consideration of the marginal thermodynamic stability of the whole crystallite, i.e., $\Delta \mathrm{F}(\mathrm{n})=$ 0 , and that of a two-dimensional critical nucleus on a smooth front. ${ }^{2}$ Experiments on a single polyethylene crystall ite grown under alternating temperatures have clearly demonstrated the existence of discontinuous 
changes in crystal thickness, ${ }^{15,16}$ which rules out the marginal thermodynamic stability of the whole crystallite as an explanation of growth thickness. The lateral front of lamellae in Figure 1 is so far from smooth that two-dimensional nucleation is not important either in the early stages or in the late stages of shish-kebab formation.

Several models have been proposed to explain why the growth thickness converges to a value just above $I_{\mathrm{min}} .{ }^{17}$ Indeed, according to the Gibbs-Thomson equation, the fact that the melting temperature is higher than the crystallization temperature means that the constant thickness of single crystal lites should be larger than $I_{\min }$. This agrees with the crystal growth condition, i.e., $\partial \Delta \mathrm{F}(\mathrm{n}) / \partial \mathrm{n}<0$. The constant lamellar thickness is quite stable in the solution without further thickening, in contrast with the growth of a single crystallite in the melt, which shows continuous thickening if there is no frictional barrier for sliding diffusion of chains in the crystallite. ${ }^{18}$ This may be attributed to the fact that any trial event of local thickening through the mechanism of sliding diffusion in the lamella with perfect regular chain folding and clean fold surface will incur a penalty of void defects in the crystalline region. ${ }^{19}$ This fact is true for the crystallites in solution but not in the melt.

At slightly lower temperatures, the density of primary nuclei on the template becomes high and upon further growth two lamellae may merge into one if they are close enough, as demonstrated in Figure 2 . An interesting phenomenon is shown in the top of Figure $2 \mathrm{a}$. The figure shows the generation of a screw dislocation that nucleates on the single-chain seed (see Figure 2b).

Our simulations demonstrate that a single chain can induce polymer epitaxy. This fact implies that there is actually no lower limit to the thickness of the central fiber in shish-kebab crystallites, supporting the scenario that chain alignment accelerates polymer nucleation. It would be possible to verify our simulation results in the experiments with powerful microscopy, since the shish-kebab morphology has already been observed on the surface of bulk polymers by atomic force microscopy $(\mathrm{AFM}),{ }^{20}$ and a single polymer chain can be stretched under the steady shear flow. ${ }^{21}$
Acknowledgment. W.H. thanks the kindly discussions with Prof. B. Lotz in Institute Charles Sadron, Prof. G. Strobl in Freiburg University, and Dr. M. Miller in Amolf. This work was supported by DSM Co. and the division of Chemical Science of the N etherlands Organization for Scientific Research (NWO). The work of the FOM Institute is part of the research program of $\mathrm{FOM}$ and is made possible by financial support from the NWO.

\section{References and Notes}

(1) Kelton, K. F. In Crystal Nucleation in Liquids and Glasses; Ehrenreich, H., Turnbull, D., Eds.; Academic Press: Boston, 1991; Vol. 45.

(2) Wunderlich, B. Macromolecular Physics; Academic Press: New York, 1976; Vol. 2, pp 1-35.

(3) Wunderlich, B. Macromolecular Physics; Academic Press: New York, 1973; Vol. 1, pp 178-379.

(4) Pennings, A.J . J . Polym. Sci., Polym. Symp. 1977, 59, 5586.

(5) McHugh, A. J . Polym. Eng. Sci. 1982, 22, 15-26.

(6) Barham, P. J .; Keller, A. J . Mater. Sci. 1985,20, 2281-2302.

(7) Keller, A.; Odell, J . A. Colloid Polym. Sci. 1985, 263, 181201.

(8) Keller, A. J. Polym. Sci. 1955, 15, 31-49.

(9) Wittmann, J. C.; Lotz, B. Prog. Polym. Sci. 1990, 15, 909948.

(10) Thierry, A.; Fillon, B.; Straupe, C.; Lotz, B.; Wittmann, J. C. Prog. Colloid Polym. Sci. 1992, 87, 28-31.

(11) Shepard, T. A.; et al. J . Polym. Sci., Part B: Polym. Phys. 1997 35, 2617-2628.

(12) Hu, W.-B. J . Chem. Phys. 1998, 109, 3686-3690.

(13) Hu, W.-B. J . Chem. Phys. 2000, 113, 3901-3908.

(14) Keller, A. Philos. Mag. 1957, 2, 1171-1175.

(15) Bassett, D. C.; Keller, A. Philos. Mag. 1961, 6, 1053-1056.

(16) Dosiere, M.; Colet, M.-Ch.; Point, J. J . In Morphology of Polymers; Sedlacek, B., Ed.; de Gruyter: Berlin, 1986; p 171.

(17) Doye, J . P. K.; Frenkel, D. J . Chem. Phys. 1999, 110, 70737086.

(18) Hu, W.-B. J . Chem. Phys. 2001,115, 4395-4401.

(19) Dreyfuss, P.; Keller, A. J . Polym. Sci., Part B 1970, 8, 253258.

(20) Hobbs, J . K.; Humphris, A. D. L.; Miles, M. J . Macromolecules 2001, 34, 5508-5519.

(21) Smith, D. E.; Dabcock, H. P.; Chu, S. Science 1999, 283, 1724-1727.

MA0255581 